\title{
Effect of alkylglycerone phosphate synthase on the expression levels of IncRNAs in glioma cells and its functional prediction
}

\author{
LEI CHEN $^{1 *}$, WEIJIAN ZHANG ${ }^{2 *}$, LIHUA HE $^{2,3}$, LI JIN $^{4}$, LIYU QIAN ${ }^{5}$ and YU ZHU ${ }^{6}$ \\ ${ }^{1}$ Department of Otolaryngology, The Second Hospital of Tianjin Medical University, Tianjin 300211; \\ ${ }^{2}$ Postgraduate School of Tianjin Medical University, Tianjin 300070; ${ }^{3}$ Department of Clinical Laboratory, \\ Affiliated Hospital of Hebei University of Engineering, Handan, Hebei 056002; ${ }^{4}$ Integrated Chinese \\ and Western Medicine School of Tianjin University of Traditional Chinese Medicine, Tianjin 301617; \\ ${ }^{5}$ Department of Tumor Surgery, The First Affiliated Hospital of Bengbu Medical College, Bengbu, Anhui \\ 233004; ${ }^{6}$ Department of Clinical Laboratory, Tianjin Haihe Hospital, Tianjin 300350, P.R. China
}

Received December 5, 2019; Accepted May 18, 2020

DOI: 10.3892/ol.2020.11927

\begin{abstract}
Alkylglycerone phosphate synthase (AGPS) is a key enzyme for ether ester synthesis and acts as an oncogene in malignant tumors. The present study aimed to investigate the effect of AGPS silencing on the expression levels of long non-coding RNAs (lncRNAs) and the co-expression with mRNAs in glioma U251 cells using microarray analysis. Furthermore, the underlying biological functions of crucial lncRNAs identified were investigated. It was discovered that in vitro U251 cell proliferation was suppressed following the genetic silencing of AGPS. Differentially expressed lncRNAs and mRNAs in U251 cells were sequenced following AGPS silencing. The results from the Gene Ontology analysis identified that the co-expressed mRNAs were mainly involved in biological processes, such as 'cellular response to hypoxia', 'extracellular matrix organization' and 'PERK-mediated unfolded protein response'. In addition, Kyoto Encyclopedia of Genes and Genomes signaling pathway enrichment analysis revealed that the co-expressed mRNAs were the most enriched in the 'AGE/RAGE signaling pathway in diabetic conditions'. Additionally, the PI3K/Akt and epidermal growth factor receptor signaling pathways serve important roles in tumor processes, for example carcinogenesis and angiogenesis. Furthermore, it was identified that the IncRNA AK093732
\end{abstract}

Correspondence to: Professor Yu Zhu, Department of Clinical Laboratory, Tianjin Haihe Hospital, 890 Jingu Road, Jinan, Tianjin 300350, P.R. China

E-mail: zhuyutj@126.com

Dr Lei Chen, Department of Otolaryngology, The Second Hospital of Tianjin Medical University, 23 Pingjiang Road, Hexi, Tianjin 300211, P.R. China

E-mail: clchenlei2008@163.com

*Contributed equally

Key words: glioma, long non-coding RNA, bioinformatics analysis served a vital role in the regulatory network and the core pathway in this network regulated by this lncRNA was discovered to be the 'Cytokine-cytokine receptor interaction'. In conclusion, the findings of the present study suggested that AGPS may affect cell proliferation and the degree of malignancy. In addition, the identified lncRNAs and their co-expressed mRNAs screened using microarrays may have significant biological effects in the occurrence, development and metastasis of glioma, and thus may be novel markers of glioma.

\section{Introduction}

Gliomas are the most common type of primary central nervous system tumor and intracranial malignant tumor, accounting for $\sim 81 \%$ of all malignant brain tumors (1). Alkylglycerone phosphate synthase (AGPS) is an important enzyme for ether ester synthesis and it has been discovered to serve an important role in the pathogenicity of cancer cells (2). It was previously reported that the overexpression of AGPS increased the survival, migratory, proliferative and invasive abilities of tumor cells, including SKOV3 ovarian cancer, 231MFP breast cancer, C8161 melanoma, PC3 prostate cancer and primary breast cancer cells $(3,4)$. Furthermore, the levels of tumor-related lipids, including ether lipids, prostaglandins and acyl phosphatides, were discovered to decrease following the inactivation of AGPS, resulting in a decrease in the cancer pathogenicity $(5,6)$. In addition, our previous study revealed a positive correlation between AGPS and the malignant potential of gliomas (7). Therefore, it was hypothesized that the overexpression of AGPS may be an important mechanism for the aggravation of gliomas.

The present study analyzed changes in the expression profiles of long non-coding RNAs (lncRNAs) and co-expressed mRNAs in glioma. IncRNAs are RNAs of $>200$ nucleotides in length, which have no protein-coding ability due to the lack of a complete open reading frame (8). lncRNAs have been identified to regulate gene expression via a variety of biological processes, demonstrating important roles in the apoptosis, proliferation, invasion and metastasis of cells (9). 
Moreover, mRNAs encode the genetic information for their corresponding protein (10). In the present study, the full-length sequence expression data for glioma cells was obtained using gene chip technology, and lncRNA and mRNA expression profiles from glioma cells were analyzed. In addition, their functions were predicted using bioinformatical methods, which provided a theoretical basis for studying the molecular mechanisms of the AGPS-induced malignancy, migration and invasiveness of glioma.

\section{Materials and methods}

Cell culture and AGPS short hairpin (sh)RNA lentivirus infection. U251 cells were obtained from the Cell Resource Center of the Chinese Academy of Medical Sciences. Cells were cultured in RPMI-1640 medium (Corning Life Sciences), supplemented with 10\% FBS (Corning Life Sciences), and maintained in a $\mathrm{CO}_{2}$-controlled incubator at a constant temperature of $37^{\circ} \mathrm{C}$.

For transfection, $2 \times 10^{5} \mathrm{U} 251$ cells were plated into a six-well plate with $2 \mathrm{ml}$ medium/well the day before transfection and cultured at $37^{\circ} \mathrm{C}$ for $24 \mathrm{~h}$ to reach $60-80 \%$ confluence on the second day. AGPS shRNA lentiviral particles (shR-AGPS-1 and -2 groups; Santa Cruz Biotechnology, Inc.) or empty lentiviral particles used as a negative control (control group; Santa Cruz Biotechnology, Inc.) stably integrated cell lines were established following infection with $8 \mu \mathrm{g} / \mathrm{ml}$ lentiviruses of U251 cells using $5 \mu \mathrm{g} / \mathrm{ml}$ polybrene-containing RPMI-1640 medium. After infecting the cells at $37^{\circ} \mathrm{C}$ for $24 \mathrm{~h}$, the medium was replaced with $2 \mathrm{ml}$ RPMI-1640 medium without polybrene, and the cells were further incubated at $37^{\circ} \mathrm{C}$ for $72 \mathrm{~h}$. Cell morphology was observed under an inverted light microscope (magnification, x100 and x200).

Monoclonal cell selection. Following infection and further cell culture for $72 \mathrm{~h}, 1 \mathrm{mg} / \mathrm{ml}$ puromycin ( $5 \mu \mathrm{l}$; Beyotime Institute of Biotechnology) was added in the RPMI-1640 medium to the cells at $80 \%$ confluence. The RPMI-1640 medium with puromycin was refreshed every 3 days for 12 days. The cells in each group were separately diluted in RPMI-1640 medium via serial dilution to produce a single cell suspension with a density of $1 \times 10^{3}$ cells $/ \mathrm{ml}$, which were subsequently plated into the first row of wells in a 96 -well plate at $0.2 \mathrm{ml} /$ well . From those first wells, $0.1 \mathrm{ml}$ cell solution was inoculated into the second row and the process was then repeated until the 8th row, with the goal of ultimately obtaining wells containing single cells. Isolated cells were cultured at $37^{\circ} \mathrm{C}$ for 1 week in complete medium to obtain monoclonal cell lines.

Cell proliferation assay. In total, $3 \times 10^{3}$ cells/well were seeded into a 96 -well plate and cultured at $37^{\circ} \mathrm{C}$ for 24,48 and $72 \mathrm{~h}$. Cell proliferation was analyzed using a BrdU cell proliferation ELISA kit (cat. no. ab126556; Abcam), according to the manufacturer's protocol. Briefly, at each time point, $20 \mu \mathrm{l} \mathrm{BrdU}$ label was added and incubated at $37^{\circ} \mathrm{C}$ for $12 \mathrm{~h}$. Following the incubation, the cells were fixed with $3.7 \%$ formaldehyde diluted in PBS at room temperature for $30 \mathrm{~min}$ and then washed three times with PBS. Then, $100 \mu \mathrm{l}$ anti-BrdU monoclonal detector antibody (1:2,000; supplied in the BrdU cell proliferation kit) was incubated with the cells for $1 \mathrm{~h}$ at room temperature. Following the incubation, each well was washed three times using PBS and $100 \mu 1$ horseradish peroxidase (HRP)-conjugated goat anti-mouse IgG antibody (1:2,000; supplied in the BrdU cell proliferation kit) was added and incubated for $30 \mathrm{~min}$ at room temperature. The wells were washed three times with PBS and then, $100 \mu 1$ 3,3,5',5'-Tetramethyl benzidine peroxidase substrate was added and incubated for $30 \mathrm{~min}$ at room temperature in the dark. Finally, $100 \mu 1$ stop solution (supplied in the BrdU cell proliferation kit) was added and the optical density value was measured every $24 \mathrm{~h}$ using the Multiskan ${ }^{\mathrm{TM}}$ Spectrum microplate photometer (Thermo Fisher Scientific, Inc.) at $450 \mathrm{~nm}$.

Western blotting. The selected stable U251 cell line $\left(2 \times 10^{5}\right.$ cells/well) was plated into a six-well plate and after $24 \mathrm{~h}$, total protein was extracted using $300 \mu \mathrm{l} /$ well RIPA lysis buffer (Beyotime Institute of Biotechnology). Cells were gently agitated at $4{ }^{\circ} \mathrm{C}$ for $30 \mathrm{~min}$ and then the cell lysate was transferred to a $1.5 \mathrm{ml}$ centrifuge tube for centrifugation at $10,000 \mathrm{x} \mathrm{g}$ for $10 \mathrm{~min}$ at $4^{\circ} \mathrm{C}$. Total protein was quantified using a bicinchoninic acid assay, and $50 \mu \mathrm{g}$ protein/lane was separated via $10 \%$ SDS-PAGE. The separated proteins were subsequently transferred onto a PVDF membrane and the membrane was blocked with $5 \%$ milk at room temperature for $2 \mathrm{~h}$. The membrane was cut around the position of the protein according to the molecular size and then it was incubated with an anti-AGPS antibody (1:1,000; cat. no. sc-374201; Santa Cruz Biotechnology, Inc.) or an anti- $\beta$-tubulin antibody (1:1,000; cat. no. SRP01044; Saierbio, LLC) at $4^{\circ} \mathrm{C}$ overnight. Following the primary antibody incubation, the membranes were washed 4 times for 5 min each with TBS-Tween (TBST; $0.5 \%$ Tween 20) solution and incubated with a HRP-conjugated goat anti-mouse IgG antibody (1:1,000; cat. no. SRPGAM001; Saierbio, LLC) or an HRP-conjugated goat anti-rabbit IgG antibody (1:1,000; cat. no. SRPGAR001; Saierbio, LLC) at room temperature for $1.5 \mathrm{~h}$. The membranes were then washed and shaken in TBST solution 4 times for 5 min each. Total protein was visualized using a Western Lightning $^{\mathrm{TM}}$ chemiluminescent agent (PerkinElmer, Inc.). The protein expression levels were analyzed using ImageJ Software v1.52 (National Institutes of Health) and the gray value for the AGPS band in each sample was normalized to the corresponding $\beta$-tubulin band. The control group was set at a standard value of 1 and a histogram was generated to compare all groups.

Screening to identify differentially expressed lncRNAs and co-expressed mRNAs. Total RNA was extracted from cells using the RNAeasy ${ }^{\mathrm{TM}}$ Animal RNA Isolation kit according to the manufacturer's protocol (Beyotime Institute of Biotechnology), and the QuantiTect Whole Transcriptome kit (Qiagen China Co., Ltd.) was used for library construction and amplification according to the manufacturer's protocol. The GeneChip Scanner 3000 System (Affymetrix; Thermo Fisher Scientific, Inc.) was used to detect the expression levels of IncRNA and the profile of co-expressed mRNAs using the Affymetrix Clariom D array in each group of cells. The control group and the two experimental groups were compared to screen differentially expressed lncRNAs and co-expressed mRNAs. The screening was analyzed using the free online 
Table I. Primer sequences used for the reverse transcription-quantitative PCR.

Gene $\quad$ Primer sequence $\left(5^{\prime} \rightarrow 3^{\prime}\right)$

COL1A2 F: GTGCGATGACGTGATCTGTGA

R: GTTTCTTGGTCGGTGGGTG

COL6A3 F: TCTGTTCCTCTTTGACGGCT

R: CCACCTTGACATCATCGCTG

THBS1 F: GCTCCAGCTCTACCAATGTCCT

R: TTGTGGCCGATGTAGTTAGTGC

FN1 F: CAGTGGGAGACCTCGAGAA

R: TCCCTCGGAACATCAGAAAC

SPP1 F: TTCTCAGCCAAACGCCGA

R: GGTAGGTACATCTTTAGTGCTGCTT

ITGA11 F: GTATTTGTAGGTTTTTTTGGATTGATTGT

R: ATCCCTATTTAAAATACACACAAAAATTTC

VEGFA F: GAGGGCAGAATCATCACGAAG

R: TGTGCTGTAGGAAGCTCATCTCTC

IGF1R F: GTGGAGACAGGGGCTTTTATT

R: CTCCAGCCTCCTTAGATCACA

EGFR F: CCTATGTGCAGAGGAATTATGATCTTT

R: CCACTGTGTTGAGGGCAAT

TLN2 F: CTGAGGCTCTTTTCACAGCA

R: CTCATCTCATCTGCCAAGCA

PXN F: GCCCCTCTCAGAGCCTTTTC

R: GCAGCTACTGAGGTCACAGC

AKT3 F: CCACAGGTCGCTACTATGCC

R: ACAGCCCGAAGTCCGTTATC

PIK3CA F: CATCATTTGCTCCAAACTGACCA

R: CCTATGCAATCGGTCTTTGCC

JUN F: AAGTGAAAACCTTGAAAGCTCAG

R: TTAACGTGGTTCATGACTTTCTG

SHC3 F: AAAAAGCTTATGAGTGCCACCAGGAAGA GCCGG

FGF2 F: GAGAAGAGCGACCCTCACA

R: TAGCTTTCTGCCCAGGTCC

ANGPT2 F: ACAGCAGAATGCAGTACAGAACCAGACG

R: CAAGTCTCGTGGTCTGATTTAATACTTGG GCT

CSF3 F: CTGCTCTAGTGGACACACAAATG R: TTTCTCCGGACTAGGCTTTG

IL7R F: AACCCCGTCTCCACTGAAAA R: GAGTCTTGCTTTGTTGCCCA

PRKAA2 F: GAAGATCGGACACTACGTGCT R: AACTGCCACTTTATGGCCTG

DDIT4 F: GGACCAAGTGTGTTTGTTGTTTG R: CACCCACCCCTTCCTACTCTT

HSP90B1 F: AAATCATTTTCAAAGGAAAGTGATGACCC R: CATCAAACAGACCACGTGGAGGAG

CREB5 F: ATTGACTCACCACCCTGCTG R: GCATGAAGGTGGGAATGGGA

TLR3 F: GCTCTGGAAACACGCAAACC R: CTCGTCAAAGCCGTTGGACT

IL1B F: GGACAGGATATGGAGCAACAAGTGG R: TTCAACACGCAGGACAGGTACAGAT
Table I. Continued.

\begin{tabular}{ll}
\hline Gene & \multicolumn{1}{c}{ Primer sequence $\left(5^{\prime} \rightarrow 3^{\prime}\right)$} \\
\hline CXCL8 & F: TCAGAGACAGCAGAGCACAC \\
& R: ACACAGTGAGATGGTTCCTTCC \\
PTGS2 & F: ATTGTACCCGGACAGGATTCTATG \\
& R: TTTGGAGTGGGTTTCAGAAATAATT \\
SDC4 & F: ACCAGACGATGAGGATGTAGTG \\
& R: AAGGGATGGACAACTTCAGGG \\
$\beta$-actin & F: CTACAATGAGCTGCGTGTG \\
& R: AAGGAAGGCTGGAAGAGTGC
\end{tabular}

F, forward; R, reverse.

platform OmicShare tools (www.omicshare.com/tools), and the criteria were fold-change $(\mid \mathrm{FCl})>1.2, \mathrm{P}<0.05$ and a false discovery rate $<0.05$.

Reverse transcription-quantitative PCR (RT-qPCR). Total RNA was extracted from cells using QIAzol reagent (Qiagen, Inc.) and reverse transcribed at $50^{\circ} \mathrm{C}$ for $15 \mathrm{~min}$ using the BeyoFast $^{\mathrm{TM}}$ Probe One-Step qRT-PCR kit (Beyotime Institute of Biotechnology), according to the manufacturer's protocol. To determine the expression levels of mRNAs, qPCR was subsequently performed using SYBR Green I dye (Takara Biotechnology Co., Ltd.) on a 7500 Real-Time PCR system (Applied Biosystems; Thermo Fisher Scientific, Inc.). The sequences of the primers used for the qPCR are listed in Table I. The following thermocycling conditions were used for the qPCR: Initial denaturation at $95^{\circ} \mathrm{C}$ for $10 \mathrm{~min}$, followed by 40 cycles at $95^{\circ} \mathrm{C}$ for $15 \mathrm{sec}$ and $60^{\circ} \mathrm{C}$ for $60 \mathrm{sec}$, and a final extension step at $72^{\circ} \mathrm{C}$ for $5 \mathrm{~min}$. The expression levels of mRNAs were quantified using the $2^{-\Delta \Delta C q}$ method (11) and normalized to the internal loading control $\beta$-actin.

Functional prediction of IncRNA and mRNA co-expression. The mRNAs co-expressed with the lncRNAs were subjected to functional term enrichment analysis using the Gene Ontology (GO) resource (http://geneontology.org), functional annotation of the differentially expressed genes and Venn diagrams were performed using free online platform OmicShare tools (www.omicshare.com/tools). Signaling pathway enrichment analysis was also performed on the co-expressed mRNAs using the Kyoto Encyclopedia of Genes and Genomes (KEGG) database (www.genome.jp) and the signaling pathways associated with the differentially expressed mRNAs were identified. Subsequently, Degree values were calculated using the OmicShare tools 3.0, and pathway networks and global signal transduction networks of co-expressed lncRNAs and mRNAs in AGPS-silenced U251 cells were constructed using the OmicShare tools with more connections there were, the higher the Degree. The differentially co-expressed lncRNAs and mRNAs predicted by GO functional term for biological process (BP) and KEGG signaling pathway enrichment analysis were used to determine the function of the unknown IncRNAs. The threshold of the Degree value was 1 . 


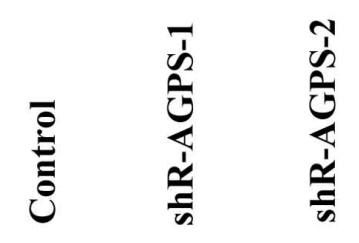

AGPS

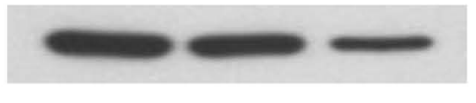

及-tubulin

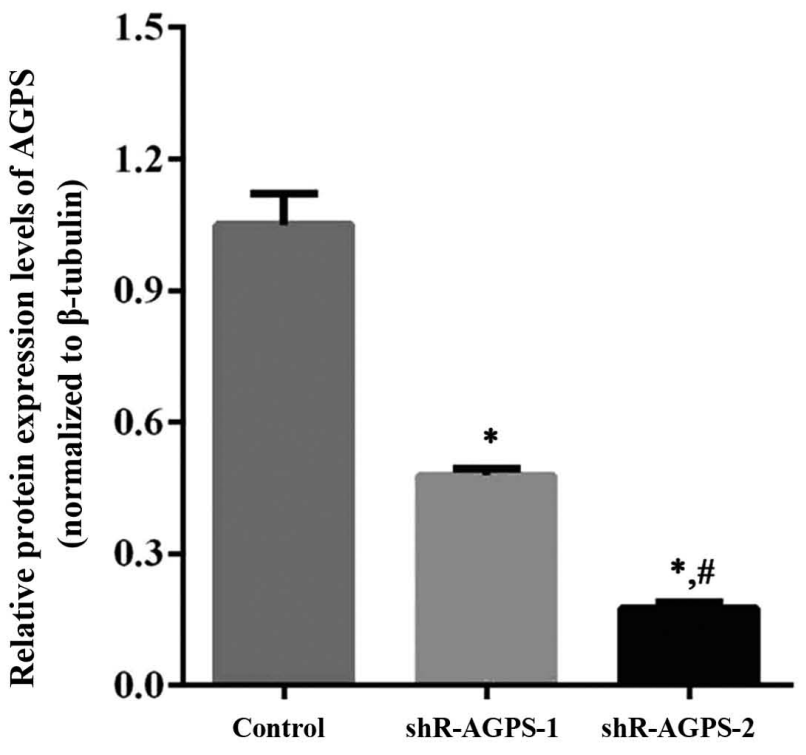

Figure 1. Effect of AGPS silencing on the expression levels of AGPS in glioma cells. Western blotting identified significantly downregulated expression levels of AGPS in AGPS-silenced U251 cells. $n=3$ "P $<0.05$ vs. control; " $\mathrm{P}<0.05$ vs. shR-AGPS-1 group. shR, short hairpin RNA; AGPS, alkylglycerone phosphate synthase.

Statistical analysis. Statistical analysis was performed using SPSS version 11 software (SPSS, Inc.). Statistical differences were determined using a one-way ANOVA, followed by a Tukey's post hoc test for multiple group comparisons. $\mathrm{P}<0.05$ was considered to indicate a statistically significant difference. A $\log 10$ (P-value $)>2$ was considered to indicate a significant threshold for GO analysis.

\section{Results}

Effect of AGPS shRNA interference on AGPS expression levels in U251 cells. Two shR-AGPS lentiviral plasmids were transfected into U251 cells to construct stable AGPS-silenced U251 cell lines; negative control lentiviruses transfected into U251 cells were used as the control. In total, two groups (shR-AGPS-1 and shR-AGPS-2 group) of AGPS-silenced cell lines were established by monoclonal selection, and the AGPS expression levels in these shR-AGPS-1 and shR-AGPS-2 groups were significantly downregulated compared with the control group (Fig. 1). Furthermore, the expression levels of AGPS in the shR-AGPS-2 group were significantly downregulated compared with the shR-AGPS-1 group (Fig. 1).

Effect of AGPS silencing on the morphology of U251 cells. After silencing the expression levels of AGPS in U251 cells, the proliferation of each group was observed under a microscope at $x 100$ and $\times 200$ magnification. The cell morphology of the shR-AGPS-1 and shR-AGPS-2 groups was observed (Fig. 2A). In addition, the proliferative ability in the shR-AGPS-2 group was significantly decreased compared with that in the shR-AGPS- 1 and control groups after 48 and $72 \mathrm{~h}$ (Fig. 2B).Cluster analysis and regulation of differentially expressed lncRNAs and mRNAs. In the heatmap, the identified downregulated lncRNAs (107 for shR-AGPS-1 vs. control, 231 for shR-AGPS-2 vs. control, and 134 for shR-AGPS-1 vs. shR-AGPS-2) and mRNAs (61 for shR-AGPS-1 vs. control, 344 for shR-AGPS-2 vs. control,
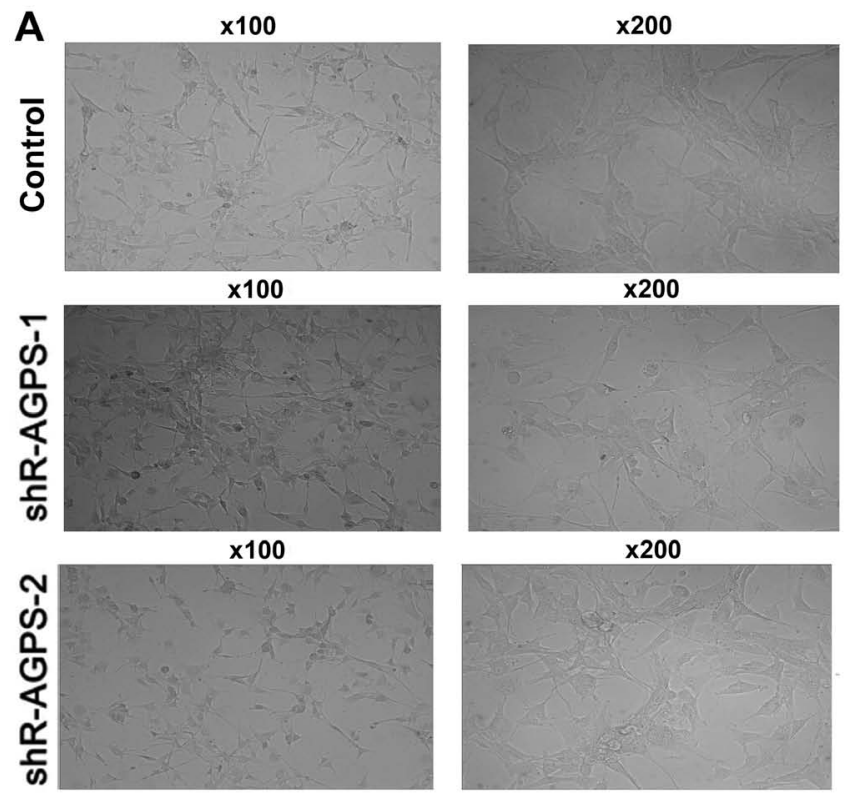

B

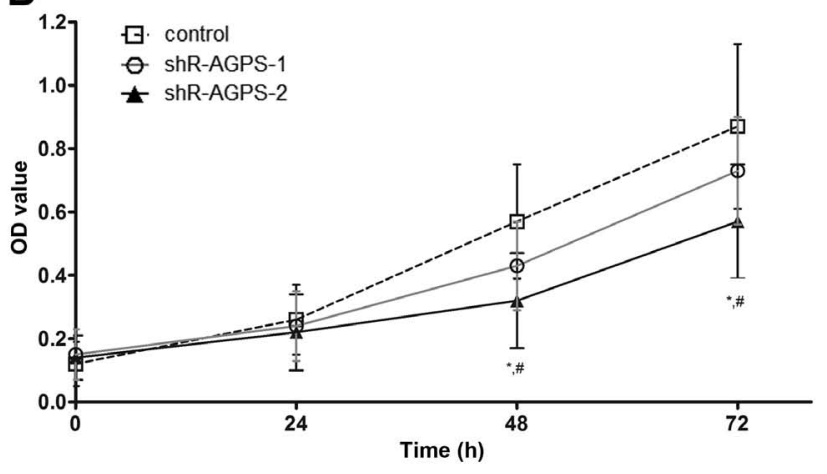

Figure 2. Effect of AGPS silencing on the cellular morphology of U251 cells. (A) Morphological observation under the microscope in AGPS-silenced U251 cells. Magnification, x100 or x200. (B) BrdU assay results revealed a significant inhibition over the proliferation of AGPS-silenced U251 cells. $\mathrm{n}=10 .{ }^{*} \mathrm{P}<0.05$ shR-2 vs. shR-1, ${ }^{\#} \mathrm{P}<0.05$ shR-1 vs. control. shR, short hairpin RNA; AGPS, alkylglycerone phosphate synthase; OD, optical density. 
Table II. Top 40 differentially expressed lncRNAs in AGPS-silenced human glioma U251 cells.

A, Top 40 differentially expressed lncRNAs in the shR-AGPS-1 vs. control groups

\begin{tabular}{|c|c|c|c|}
\hline lncRNA & Upregulated FC & $\operatorname{lncRNA}$ & Downregulated FC \\
\hline LINC00837 & 1.79 & CR604135 & 1.32 \\
\hline CR626472 & 1.49 & CTC-436K 13.3 & 1.76 \\
\hline ERI3-IT1 & 1.48 & AK129941 & 1.64 \\
\hline linc-AKR1B10-2 & 1.44 & MIR548AA2 & 1.58 \\
\hline AF344193 & 1.44 & linc-FCGR1B-7 & 1.57 \\
\hline LINC01079 & 1.41 & AK098597 & 1.42 \\
\hline XXbac-B33L19.4 & 1.41 & AF086041 & 1.40 \\
\hline ENST00000551732 & 1.40 & linc-LOC388630-3 & 1.40 \\
\hline $\mathrm{AC} 008440.10$ & 1.40 & linc_luo_384 & 1.40 \\
\hline linc_luo_541 & 1.39 & linc-SEC23B & 1.39 \\
\hline linc-TMEM169-2 & 1.35 & linc-CNTN3-3 & 1.39 \\
\hline linc-MAGEA1-1 & 1.35 & linc-TEKT4-1 & 1.39 \\
\hline AF087965 & 1.34 & uc003srb.1 & 1.38 \\
\hline LOC 101928354 & 1.34 & BX641068 & 1.38 \\
\hline FENDRR & 1.34 & linc_luo_692 & 1.36 \\
\hline CR618740 & 1.33 & uc011edo.1 & 1.35 \\
\hline linc-ZFP42-2 & 1.33 & LINC00973 & 1.34 \\
\hline linc-B4GALT1 & 1.32 & FAM138B & 1.34 \\
\hline ВC047484 & 1.31 & LUCAT1 & 1.33 \\
\hline AK054755 & 1.29 & linc-ERG-8 & 1.33 \\
\hline
\end{tabular}

B, Top 40 differentially expressed lncRNAs in the shR-AGPS-2 vs. control groups

\begin{tabular}{|c|c|c|c|}
\hline lncRNA & Upregulated FC & lncRNA & Downregulated FC \\
\hline LINC00837 & 2.95 & CR618823 & 2.59 \\
\hline FENDRR & 2.21 & MIR548AA2 & 2.58 \\
\hline linc-U2AF1-1 & 1.71 & LINC00973 & 2.42 \\
\hline CTD-2377D24.4 & 1.66 & linc_luo_1251 & 2.11 \\
\hline LOC 100506532 & 1.56 & LINC00263 & 2.01 \\
\hline FENDRR & 1.55 & linc-JAK1-1 & 1.95 \\
\hline CR624806 & 1.55 & $\mathrm{BC} 013423$ & 1.88 \\
\hline AK130416 & 1.50 & linc-COL1A2-2 & 1.87 \\
\hline linc-ARHGAP11B-2 & 1.47 & linc_luo_1846 & 1.85 \\
\hline uc004cic. 2 & 1.46 & linc-WDR7-7 & 1.83 \\
\hline AF344193 & 1.46 & AC003092.1 & 1.82 \\
\hline AF147353 & 1.45 & uc001tkz.2 & 1.81 \\
\hline linc-UMODL1-5 & 1.44 & linc_luo_1223 & 1.79 \\
\hline linc-AKR1B10-2 & 1.44 & AL110176 & 1.78 \\
\hline linc-SLC16A7-1 & 1.42 & LUCAT1 & 1.76 \\
\hline ERI3-IT1 & 1.42 & СТC-436K 13.3 & 1.76 \\
\hline linc-CDH11-5 & 1.42 & uc003flo.2 & 1.7 \\
\hline AL133249.1 & 1.4 & uc001gla.1 & 1.69 \\
\hline ENST551732 & 1.4 & FAM138B & 1.66 \\
\hline AC008440.10 & 1.4 & uc003cpd.1 & 1.65 \\
\hline
\end{tabular}


Table II. Continued.

C, Top 40 differentially expressed lncRNAs in the shR-AGPS-1 vs. shR-AGPS-2 groups

\begin{tabular}{lclc}
\hline IncRNA & Upregulated FC & \multicolumn{1}{c}{ lncRNA } & Downregulated FC \\
\hline FENDRR & 1.65 & CR618823 & 1.97 \\
LINC00837 & 1.65 & LINC00973 & 1.80 \\
CTD-2377D24.4 & 1.62 & BC013423 & 1.79 \\
linc-U2AF1-1 & 1.61 & LINC00263 & 1.70 \\
linc-LRRC8D-1 & 1.53 & AL110176 & 1.69 \\
linc-ACVR2B & 1.47 & linc_luo_1223 & 1.64 \\
linc-GPATCH2-4 & 1.46 & MIR548AA2 & 1.63 \\
AF147353 & 1.45 & linc-WDR7-7 & 1.62 \\
linc-FBRSL1-3 & 1.44 & linc_luo_1251 & 1.61 \\
LINC01314 & 1.44 & uc002axu.2 & 1.58 \\
linc-UMODL1-5 & 1.44 & uc001tkz.2 & 1.57 \\
linc-SLC16A7-1 & 1.42 & linc_luo_888 & 1.53 \\
linc-RREB1-2 & 1.42 & AX721161 & 1.53 \\
linc-CDH11-5 & 1.42 & linc-COL1A2-2 & 1.53 \\
linc-PELO-3 & 1.40 & linc-PTPRQ-4 & 1.50 \\
linc-GADD45G-1 & 1.40 & CR598627 & 1.50 \\
linc-COX4NB-1 & 1.39 & AL833129 & 1.49 \\
RP11-339N8.1 & 1.38 & CR603hoi.2 & 1.49 \\
linc-SIPA1L1 & 1.38 & uc003cpd.1 & 1.49 \\
U84508 & 1.37 & & 1.49 \\
\hline
\end{tabular}

lncRNA, long non-coding RNA; FC, fold change; shR, short hairpin RNA; AGPS, alkylglycerone phosphate synthase.
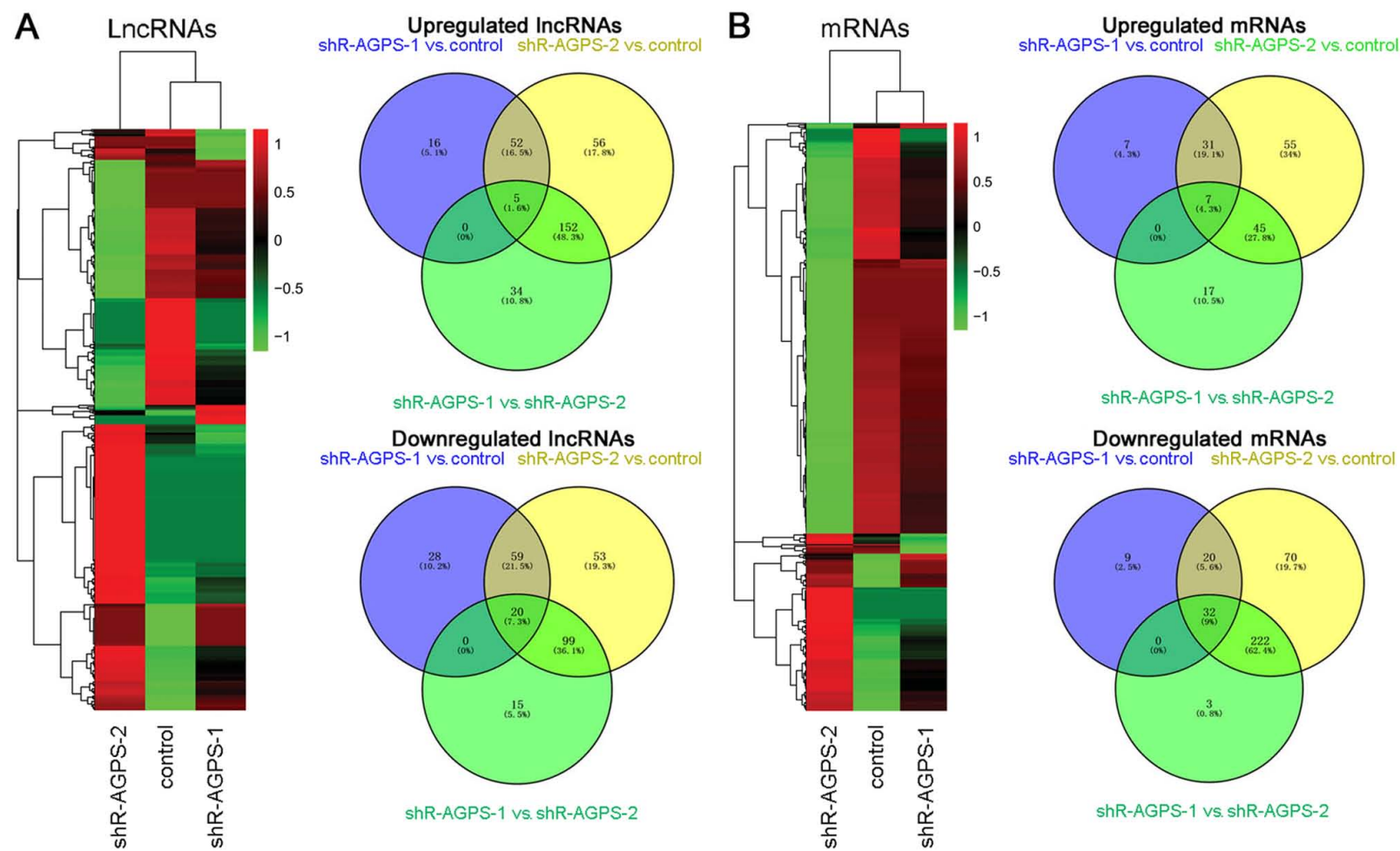

shR-AGPS-1 vs. shR-AGPS-2

Figure 3. Distribution of differentially expressed lncRNAs and mRNAs identified in AGPS-silenced glioma cells. Heatmap and Venn diagrams of differentially expressed (A) lncRNAs and (B) mRNAs in AGPS-silenced U251 cells. The differential expression of lncRNAs and mRNAs were depicted in the heatmap. Green represents the downregulated expression of $1 \mathrm{ncRNAs} / \mathrm{mRNAs}$ and red represents the upregulated expression of $1 \mathrm{ncRNAs} / \mathrm{mRNAs}$. The Venn diagrams represent the shared modified genes between groups. $\mathrm{n}=3$. The scale bar indicates the $\mathrm{FC}$, and $(\mathrm{IFCl})>1.2$ was used as a threshold. shR, short hairpin RNA; AGPS, alkylglycerone phosphate synthase; lncRNA, long non-coding RNA; FC, fold-change. 
Table III. Top 40 differentially expressed mRNAs in AGPS-silenced human glioma U251 cells.

A, Top 40 differentially expressed mRNAs in the shR-AGPS-1 vs. control groups

\begin{tabular}{|c|c|c|c|}
\hline mRNA & Upregulated FC & mRNA & Downregulated FC \\
\hline OR2J2 & 1.84 & CXCL8 & 2.10 \\
\hline THBS1 & 1.58 & SLC7A11 & 1.81 \\
\hline KIR2DL5B & 1.53 & APOH & 1.56 \\
\hline LTB & 1.50 & HMGA2 & 1.53 \\
\hline PRPS1 & 1.42 & PSAT1 & 1.49 \\
\hline HIST1H3I & 1.41 & BLID & 1.45 \\
\hline INTS4 & 1.35 & ZNF404 & 1.44 \\
\hline RGCC & 1.35 & SLC38A1 & 1.43 \\
\hline MFAP4 & 1.34 & CXCL8 & 1.43 \\
\hline KRT75 & 1.33 & IGHD3-16 & 1.42 \\
\hline UNK & 1.32 & ZNF404 & 1.42 \\
\hline HES 1 & 1.32 & C3orf17 & 1.41 \\
\hline NQO1 & 1.30 & CHML & 1.41 \\
\hline FAIM2 & 1.30 & IGFBP1 & 1.39 \\
\hline HIST1H4C & 1.28 & RPE 1.35 & \\
\hline KIR3DL2 & 1.28 & OR2Z1 & 1.32 \\
\hline KRTAP5-3 & 1.27 & SLC7A5 & 1.32 \\
\hline CCL2 & 1.27 & DST 1.31 & \\
\hline HIST2H2AA4 & 1.26 & PDK3 & 1.30 \\
\hline IGKV2-40 & 1.26 & ASNS & 1.30 \\
\hline
\end{tabular}

B, Top 40 differentially expressed mRNAs in the shR-AGPS-2 vs. control groups

\begin{tabular}{|c|c|c|c|}
\hline mRNA & Upregulated FC & mRNA & Downregulated FC \\
\hline THBS1 & 2.31 & CXCL8 & 6.94 \\
\hline PRPS1 & 1.89 & AGPS & 4.53 \\
\hline RGCC & 1.79 & IGFBP1 & 4.22 \\
\hline KRT75 & 1.71 & $\mathrm{APOH}$ & 3.22 \\
\hline NQO1 & 1.67 & CXCL8 & 2.70 \\
\hline HIST1H3I & 1.67 & SLC7A11 & 2.67 \\
\hline KIR2DL5B & 1.63 & SLC38A1 & 2.45 \\
\hline CNN2 & 1.61 & PTGS2 & 2.29 \\
\hline PLEKHA6 & 1.60 & TREM1 & 2.28 \\
\hline IGKC & 1.56 & $\mathrm{SCD}$ & 2.21 \\
\hline MFAP4 & 1.56 & CHML & 2.20 \\
\hline ITGA11 & 1.55 & OGFRL1 & 2.16 \\
\hline OR10P1 & 1.51 & IL7R & 2.15 \\
\hline LTB & 1.50 & IGFBP3 & 2.12 \\
\hline TRDV1 & 1.46 & SEL1L3 & 2.09 \\
\hline CCL2 & 1.46 & HMGA2 & 2.06 \\
\hline DEPTOR & 1.46 & PSAT1 & 2.05 \\
\hline NOB1 & 1.45 & DOCK10 & 2.03 \\
\hline C1QBP & 1.43 & ROS1 & 2.03 \\
\hline KRTAP10-10 & 1.42 & PMAIP1 & 1.98 \\
\hline
\end{tabular}


Table III. Continued.

C, Top 40 differentially expressed mRNAs in the shR-AGPS-1 vs. shR-AGPS- 2 groups

\begin{tabular}{|c|c|c|c|}
\hline mRNA & Upregulated FC & mRNA & Downregulated FC \\
\hline THBS1 & 1.46 & AGPS & 3.55 \\
\hline IGHJ1 & 1.43 & CXCL8 & 3.30 \\
\hline GPR33 & 1.38 & IGFBP1 & 3.04 \\
\hline IGHD3-16 & 1.37 & OR2J2 & 2.20 \\
\hline IGKC & 1.34 & $\mathrm{APOH}$ & 2.06 \\
\hline KRTAP19-4 & 1.34 & TREM1 & 1.90 \\
\hline PLEKHA6 & 1.34 & SCD & 1.90 \\
\hline EN2 & 1.33 & CXCL8 & 1.89 \\
\hline BTNL2 & 1.33 & IGFBP3 & 1.87 \\
\hline RGCC & 1.33 & PTGS2 & 1.84 \\
\hline PRPS1 & 1.32 & OGFRL1 & 1.83 \\
\hline OR2Z1 & 1.32 & SLC38A1 & 1.71 \\
\hline VN1R4 & 1.31 & SEL1L3 & 1.70 \\
\hline OR10P1 & 1.30 & STC1 & 1.70 \\
\hline ATOH8 & 1.30 & ROS1 & 1.69 \\
\hline CNN2 & 1.30 & IL7R & 1.69 \\
\hline IGHV3OR16-9 & 1.30 & SLC2A3 & 1.66 \\
\hline OR51B6 & 1.30 & BDKRB1 & 1.65 \\
\hline DEPTOR & 1.29 & PANK3 & 1.63 \\
\hline KRT75 & 1.29 & TXNIP & 1.62 \\
\hline
\end{tabular}

FC, fold change; shR, short hairpin RNA; AGPS, alkylglycerone phosphate synthase.

and 257 for shR-AGPS-1 vs. shR-AGPS-2) are represented in green, while the identified upregulated lncRNAs ( 73 for shR-AGPS-1 vs. control, 265 for shR-AGPS-2 vs. control, and 191 for shR-AGPS-1 vs. shR-AGPS-2) and mRNAs (45 for shR-AGPS-1 vs. control, 138 for shR-AGPS-2 vs. control, and 69 for shR-AGPS-1 vs. shR-AGPS-2) are represented in red, in AGPS-silenced and control glioma cells (Fig. 3A and B). Venn diagrams were used to compare the differentially regulated lncRNAs and mRNAs in the control, shR-AGPS-1 and shR-AGPS-2 groups. There were 57 and 79 lncRNAs shared between shR-AGPS-1 vs. control and shR-AGPS-2 vs. control, 5 and 20 shared between shR-AGPS-1 vs. control and shR-AGPS-1 vs. shR-AGPS-2, 157 and 119 shared between shR-AGPS-2 vs. control and shR-AGPS-1 vs. shR-AGPS-2, and 5 and 20 shared among all three groups for upregulated and downregulated lncRNAs, respectively; additionally, there were 38 and 52 mRNAs shared between shR-AGPS-1 vs. control and shR-AGPS-2 vs. control, 7 and 32 shared between shR-AGPS-1 vs. control and shR-AGPS-1 vs. shR-AGPS-2, 52 and 254 shared between shR-AGPS-2 vs. control and shR-AGPS-1 vs. shR-AGPS-2, and 7 and 32 shared among all three groups for upregulated and downregulated mRNAs, respectively (Fig. 3A and B). The top 40 differentially expressed lncRNAs and mRNAs, such as FOXF1 adjacent non-coding developmental regulatory RNA (FENDRR) and OR2J2 (which were upregulated compared with the control group), are presented in Tables II and III. RNAs with a $(\mid \mathrm{FCl})>1.2$ were upregulated or downregulated.
Functional prediction based on IncRNA and mRNA co-expression. According to the KEGG biological function analysis, the biological functions that were significantly enriched by differentially expressed genes were mainly involved in 'Human Diseases', 'Environmental Information Processing' and 'Organismal Systems' in biological systems. The biological functions that were significantly enriched by differential gene analysis were mainly involved in the 'Infectious diseases', Signal transduction' and 'Immune system' in cellular functions (Fig. 4A).

The co-expressed mRNAs identified in the present study were subjected to signaling pathway enrichment analysis by KEGG. It was identified that significantly enriched signaling pathways for the co-expressed mRNAs included the 'AGE-RAGE signaling pathway in diabetic complications', 'Rheumatoid arthritis', 'TNF signaling pathway', 'Protein processing in endoplasmic reticulum' and 'Malaria' (Fig. 4B).

GO enrichment BP analysis of the co-expressed lncRNAs and $\mathrm{mRNAs}$ indicated that the BPs of these two regulated types of RNA were associated with 'Cellular response to hypoxia', 'Extracellular matrix organization' and 'PERK-mediated unfolded protein response' (Fig. 4C); these functions are known to mediate protein responses, cell adhesion and the positive regulation of angiogenesis (12-14).

Construction of a signal transduction and pathway network from the co-expressed lncRNAs and mRNAs. Based on the 
A

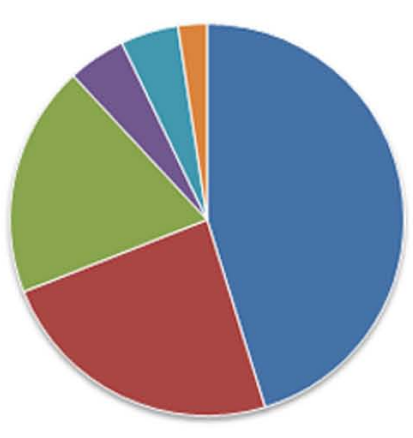

Biological systems

Human diseases

- Environmental information processing

" Organismal systems

n Cellular processes

" Metabolism

= Genetic information processing

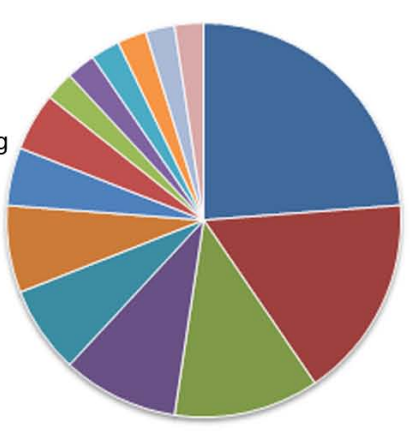

Cellular functions

- Infectious diseases

m Signal transduction

=Immune system

- Cancers

- Endocrine and metabolic diseases

w Signaling molecules and interaction

Endocrine system

= Immune diseases

= Cell growth and death

- Cellular commiunity

= Environmental adaptation

= Folding, sorting and degradation

= Lipid metabolism

= Overview

\section{B KEGG analysis}

AGE-RAGE signaling pathway in diabetic complications Rheumatoid arthritis

TNF signaling pathway

Protein processing in endoplasmic reticulum

Malaria

HIF-1 signaling pathway

Focal adhesion

Biosynthesis of amino acids

NF-kB signaling pathway

mTOR signaling pathway

Circadian rhythm

ECM-receptor interaction

PI3K-Akt signaling pathway

Central carbon metabolism in cancer

Herpes simplex infection

Chagas disease (American trypanosomiasis)

p53 signaling pathway

Insulin resistance

Staphylococcus aureus infection

Influenza $A$

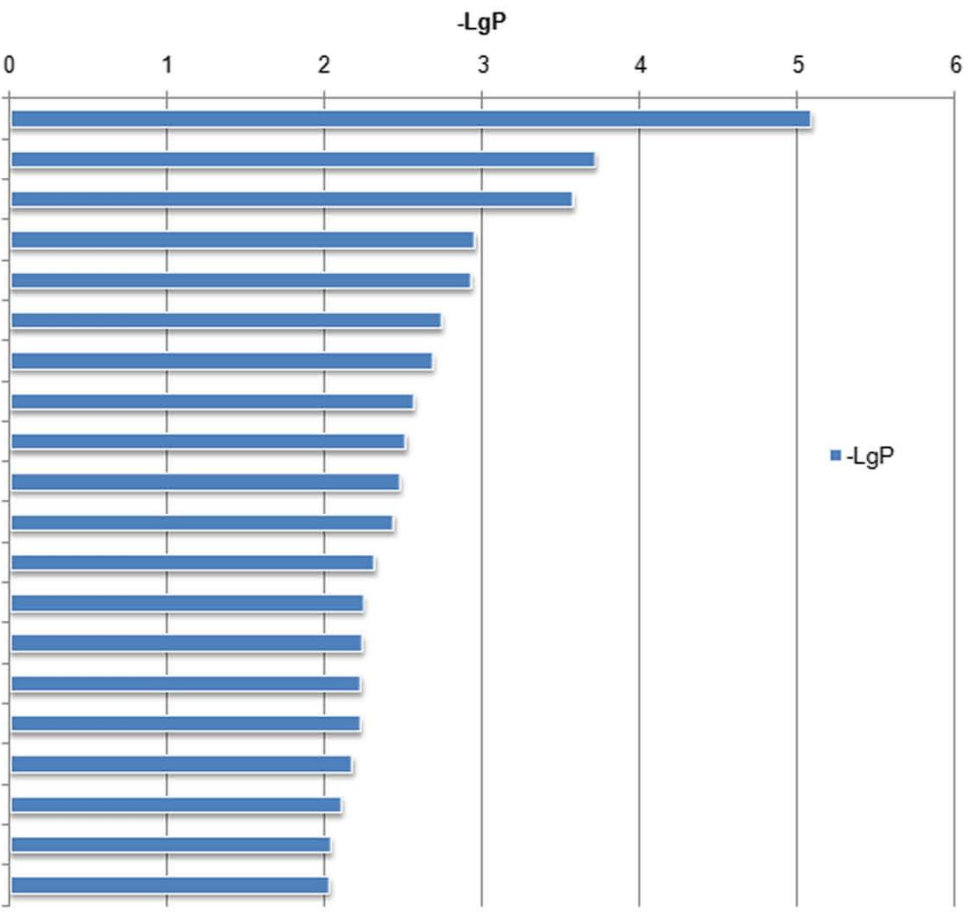

\section{C $\mathrm{GO}$ analysis}
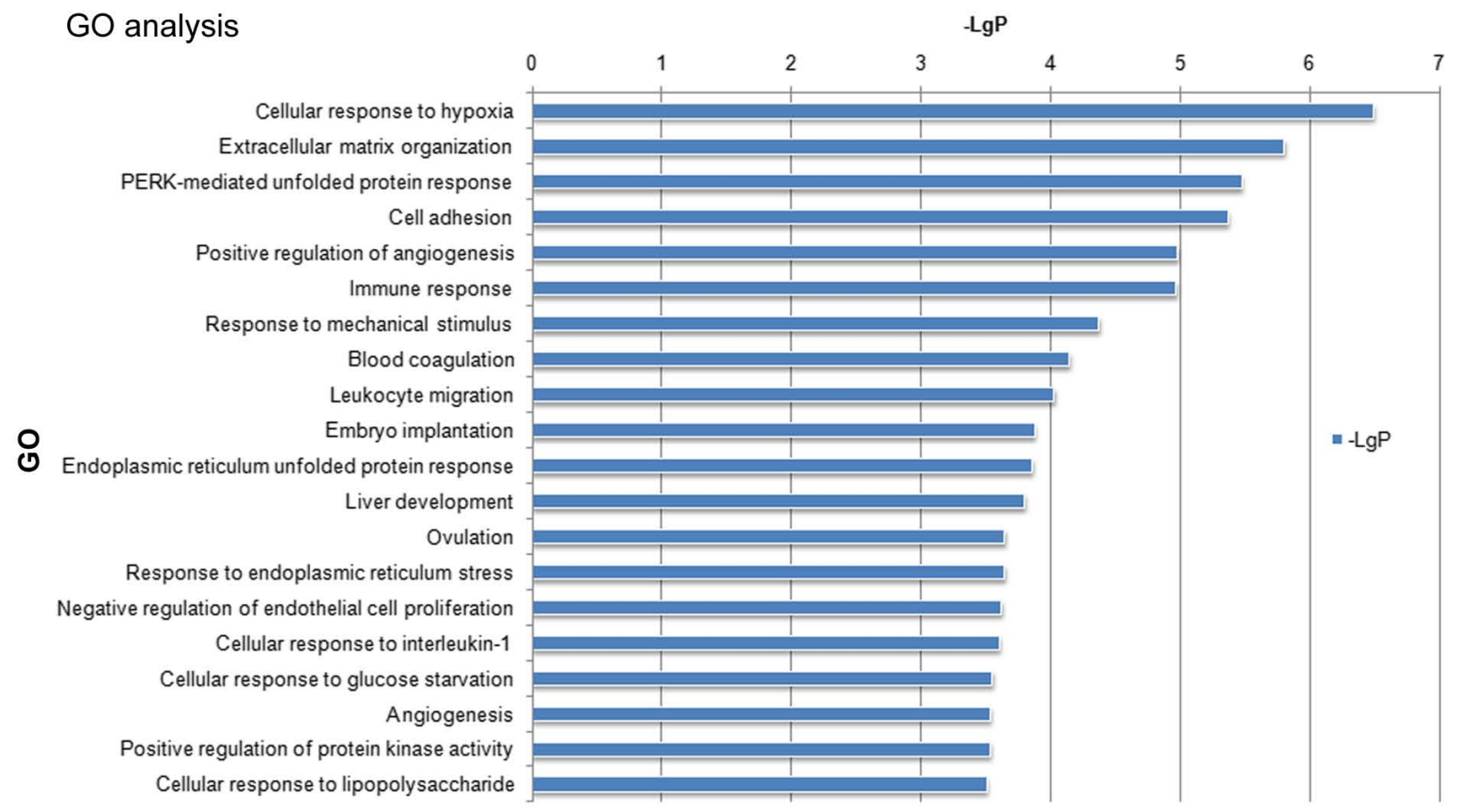

Figure 4. Functional prediction based on long non-coding RNA and mRNA co-expression in AGPS-silenced U251 cells. (A) Classification of KEGG biological functions analysis in AGPS-silenced glioma cells in Biological systems and Cellular functions. (B) Top 20 signaling pathways identified using KEGG signaling pathway enrichment analysis of AGPS-silenced U251 cells. (C) Top 20 biological process terms identified using GO enrichment analysis of AGPS-silenced U251 cells. AGPS, alkylglycerone phosphate synthase; KEGG, Kyoto Encyclopedia of Genes and Genomes; GO, Gene Ontology. 

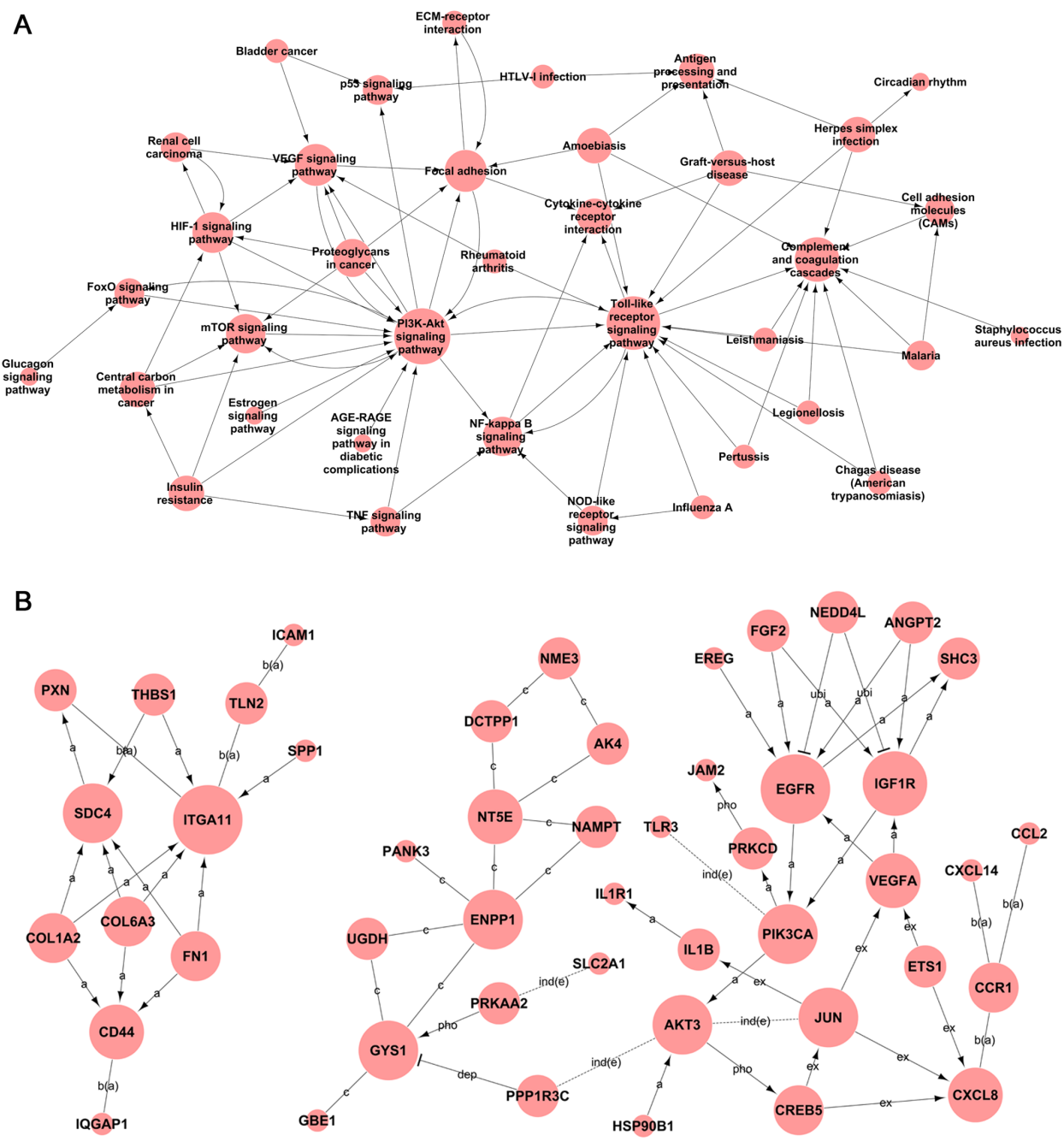

Figure 5. Signaling transduction and pathway network of co-expressed lncRNAs and mRNAs in AGPS-silenced U251 cells. (A) Pathway network of co-expressed lncRNAs and mRNAs in AGPS-silenced U251 cells. (B) Global signal transduction network of co-expressed lncRNAs and mRNAs in AGPS-silenced U251 cells. The nodes represent signaling pathways, IncRNAs and mRNAs and the arrows (or edges) represent the interaction/regulation between nodes. Ex, expression; pho, phosphorylation; a, activation; ind(e), indirect effect; b(a), binding/association; ubi, ubiquitination; dep, dephosphorylation; c, compound; s(c), state change; inh, inhibition; lncRNA, long non-coding RNA; AGPS, alkylglycerone phosphate synthase.

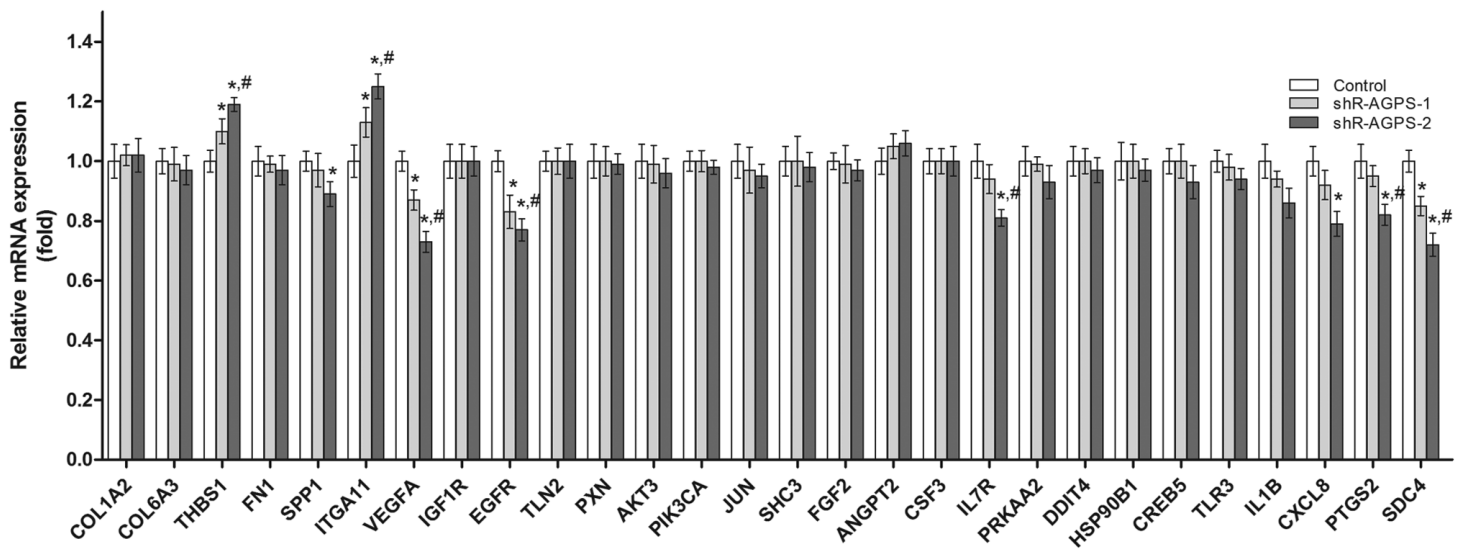

Figure 6. Effect of AGPS silencing on the expression levels of tumor-related mRNAs in glioma cells. Reverse transcription-quantitative PCR was used to analyze the mRNA expression levels of tumor-related mRNAs in AGPS-silenced U251 cells. ${ }^{*} \mathrm{P}<0.05$ vs. control group; $\mathrm{P}<0.05$ vs. shR-AGPS-1 group. COL1A2, collagen I $\alpha 2$; COL6A3, collagen $6 \alpha 3$; THBS1, thrombospondin-1; FN1, fibronectin 1; SPP1, secreted phosphoprotein 1; ITGA11, integrin subunit a11; VEGFA, vascular endothelial growth factor A; IGF1R, insulin-like growth factor 1 receptor; EGFR, epidermal growth factor receptor; TLN2, Talin 2; PXN, Paxillin; PIK3CA, phosphoinositide 3-kinase $\alpha$; SHC3, Src homology 2 domain containing transforming protein 3; FGF2, fibroblast growth factor 2; ANGPT2, angiopoietin 2; CSF3, colony stimulating factor 3; IL-7R, interleukin-7 receptor subunit $\alpha$; PRKAA2, protein kinase AMP-activated $\alpha 2$; DDIT4, DNA damage inducible transcript 4; HSP90B1, heat shock protein $90 \mathrm{kDa} \beta 1$; CREB5, cyclic AMP-responsive element-binding protein 5; TLR3, Toll-like receptor 3; IL-1B, interleukin-1 $\beta$; CXCL8, chemokine ligand 8; PTGS2, prostaglandin G/H synthase 2; SDC4, syndecan-4; shRNA, short hairpin RNA; AGPS, alkylglycerone phosphate synthase. 
Table IV. Pathway networks in alkylglycerone phosphate synthase-silenced human glioma U251 cells.

\begin{tabular}{|c|c|c|c|}
\hline Pathway name & Degree & Indegree & Outdegree \\
\hline PI3K-Akt signaling pathway & 19 & 12 & 7 \\
\hline Toll-like receptor signaling pathway & 17 & 13 & 4 \\
\hline Complement and coagulation cascades & 10 & 10 & 0 \\
\hline Focal adhesion & 8 & 5 & 3 \\
\hline VEGF signaling pathway & 8 & 6 & 2 \\
\hline HIF-1 signaling pathway & 7 & 3 & 4 \\
\hline mTOR signaling pathway & 6 & 5 & 1 \\
\hline NF-kappa B signaling pathway & 6 & 4 & 2 \\
\hline Proteoglycans in cancer & 5 & 0 & 5 \\
\hline Central carbon metabolism in cancer & 4 & 1 & 3 \\
\hline Graft-versus-host disease & 4 & 0 & 4 \\
\hline Antigen processing and presentation & 4 & 4 & 0 \\
\hline Herpes simplex infection & 4 & 0 & 4 \\
\hline Cytokine-cytokine receptor interaction & 4 & 4 & 0 \\
\hline Amoebiasis & 4 & 0 & 4 \\
\hline Insulin resistance & 4 & 0 & 4 \\
\hline TNF signaling pathway & 3 & 1 & 2 \\
\hline Renal cell carcinoma & 3 & 1 & 2 \\
\hline Cell adhesion molecules & 3 & 0 & 0 \\
\hline FoxO signaling pathway & 3 & 2 & 1 \\
\hline Malaria & 3 & 0 & 3 \\
\hline NOD-like receptor signaling pathway & 3 & 1 & 2 \\
\hline p53 signaling pathway & 3 & 3 & 0 \\
\hline Legionellosis & 2 & 0 & 2 \\
\hline Pertussis & 2 & 0 & 2 \\
\hline HTLV-I infection & 2 & 0 & 2 \\
\hline ECM-receptor interaction & 2 & 1 & 1 \\
\hline Influenza A & 2 & 0 & 2 \\
\hline Leishmaniasis & 2 & 0 & 2 \\
\hline Bladder cancer & 2 & 0 & 2 \\
\hline Chagas disease (American trypanosomiasis) & 2 & 0 & 0 \\
\hline Rheumatoid arthritis & 2 & 0 & 2 \\
\hline AGE-RAGE signaling pathway in diabetic complications & 1 & 0 & 1 \\
\hline Circadian rhythm & 1 & 1 & 0 \\
\hline Glucagon signaling pathway & 1 & 0 & 1 \\
\hline Staphylococcus aureus infection & 1 & 0 & 1 \\
\hline Estrogen signaling pathway & 1 & 0 & 1 \\
\hline
\end{tabular}

signal transduction pathway interactions identified by KEGG signaling pathway enrichment analysis, a signaling pathway network was constructed using the OmicShare tools. The network consisted of 37 nodes and 158 connections. Amongst the most connected nodes identified was the PI3K-Akt signaling pathway, the Toll-like receptor signaling pathway, the complement and coagulation cascades, focal adhesion and the vascular endothelial growth factor (VEGF) signaling pathway (Fig. 5A). The analysis of the mRNAs corresponding to the signaling pathway action network was used to construct a global signal transduction network based on the interaction between genes, proteins and compounds, and to further obtain a network of interactions between mRNAs using the OmicShare tools. The network consisted of 75 nodes and 164 connections. The epidermal growth factor receptor (EGFR), integrin $\alpha-11$ (ITGA11) and insulin-like growth factor 1 receptor (IGF1R) were the central sites in the pathway network (Fig. 5B).

Pathway network in AGPS-silenced human glioma cells. The pathway network in AGPS-silenced U251 cells is presented in Table IV. To further assess the expression levels and regulatory effect of AGPS on the pathway networks in human glioma cells, the mRNAs of important genes in the PI3K/Akt, Toll-like receptor, focal adhesion and VEGF signaling pathways were analyzed using RT-qPCR. Compared with the control cells, significantly upregulated expression levels of 
thrombospondin-1 (THBS1) and ITGA11, and significantly downregulated expression levels of secreted phosphoprotein 1 (SPP1), VEGFA, EGFR, interleukin-7 receptor subunit $\alpha$ (IL7R), interleukin-8 (CXCL8), prostaglandin G/H synthase 2 (PTGS2) and syndecan-4 (SDC4), were identified in the shR-AGPS-transfected cells (Fig. 6).

IncRNA regulation pathway network. A IncRNA regulatory network was constructed using the OmicShare tools. Moreover, 979 connections were identified between the nodes. The Degree of the IncRNA and its target pathway was calculated by OmicShare tools 3.0 according to the connection around the IncRNA and its target pathway, and the more connections there were, the higher the Degree. Table V shows that the IncRNA with the highest Degree was AK093732. To analyze the importance of each pathway in the network, it was identified that the core signal transduction pathway regulated by differentially expressed lncRNAs was cytokine-cytokine receptor interaction (Fig. 7). The top 20 lncRNA-target pathway networks in AGPS-silenced U251 cells are presented in Table V.

\section{Discussion}

AGPS is an enzyme that converts acylglycerol-3-phosphate to alkylglycerol-3-phosphate, which is a necessary step to generate all ether lipids (4). Cancer cells are known to metabolize lipids in a manner that is different from normal cells (15). In addition, the content of ether lipids in tumors has been discovered to be higher compared with healthy tissues (16). Moreover, the levels of ether lipids in malignant tumors were found to be increased compared with non-invasive tumors, and AGPS expression levels were also observed to be different in cancer cells (5-7). Tumorigenicity is known to be caused by invasive cancer cells (17), which is consistent with the present findings. In the present study, it was identified that, compared with the control group, AGPS silencing suppressed the proliferation of U251 cells.

The effect of AGPS on the pathway network was analyzed in human glioma cells by sequencing and bioinformatics analysis, and it was discovered that AGPS silencing regulated tumor-related signaling pathways, such as the PI3K/Akt, Toll-like receptor, focal adhesion and VEGF signaling pathways. These aforementioned results were further validated by RT-qPCR, where significantly upregulated expression levels of THBS1 and ITGA11, and downregulated expression levels of SPP1, VEGFA, EGFR, IL7R, CXCL8, PTGS2 and SDC4, were all identified; all of these are crucial genes involved in the aforementioned signaling pathways, and have been found to serve roles in tumor metastasis and angiogenesis (18-23).

IncRNAs were once considered to be a noisy byproduct of the transcription process (24). However, previous studies have reported that lncRNAs serve an important role at the RNA level in regulating cell proliferation, differentiation, the maintenance of pluripotent stem cells and cancer pathogenesis $(25,26)$. The present study aimed to identify the lncRNAs associated with glioma, and subsequently used bioinformatics to analyze the biological functions and signal transduction pathways involved in the regulation of the IncRNAs identified. Furthermore, a regulatory network of IncRNAs in relation
Table V. Top 20 lncRNA-target pathway networks in alkylglycerone phosphate synthase-silenced U251 cells.

\begin{tabular}{lc} 
A, Pathway & Degree \\
\hline Cytokine-cytokine receptor interaction & 49 \\
PI3K-Akt signaling pathway & 45 \\
Cell adhesion molecules (CAMs) & 40 \\
AGE-RAGE signaling pathway & 36 \\
p53 signaling pathway & 36 \\
Rheumatoid arthritis & 36 \\
NF-kappa B signaling pathway & 34 \\
Herpes simplex infection & 33 \\
Malaria & 33 \\
TNF signaling pathway & 30 \\
Glucagon signaling pathway & 29 \\
Focal adhesion & 28 \\
HTLV-I infection & 28 \\
Influenza A & 27 \\
Bladder cancer & 26 \\
Chagas disease (American trypanosomiasis) & 26 \\
Central carbon metabolism in cancer & 25 \\
Toll-like receptor signaling pathway & 24 \\
HIF-1 signaling pathway & 22 \\
Leishmaniasis & 22
\end{tabular}

B, lncRNA Degree

\begin{tabular}{ll}
\hline AK093732 & 34 \\
LINC01384 & 27 \\
FENDRR & 25 \\
uc011bsz.1 & 25 \\
linc-PTPRN2 & 24 \\
linc-U2AF1-1 & 24 \\
RP11-221N13.3 & 24 \\
BC042023 & 23 \\
BC043357 & 23 \\
linc_luo_1251 & 23 \\
linc_luo_35 & 23 \\
linc_luo_1223 & 22 \\
LINC00973 & 22 \\
linc-ANKRD20A1-20 & 22 \\
linc-NBPF15-1 & 22 \\
uc003flo.2 & 22 \\
linc-ANKRD20A1-14 & 21 \\
linc-DUSP10-7 & 21 \\
linc-FOXB2-3 & 21 \\
linc-WISP3-2 & 21
\end{tabular}

lncRNA, long non-coding RNA.

to signal transduction pathways was constructed to identify lncRNAs in a pivotal position. For example, FENDRR is an endothelial cell gene critical for vascular development, and it was previously reported that FENDRR overexpression 


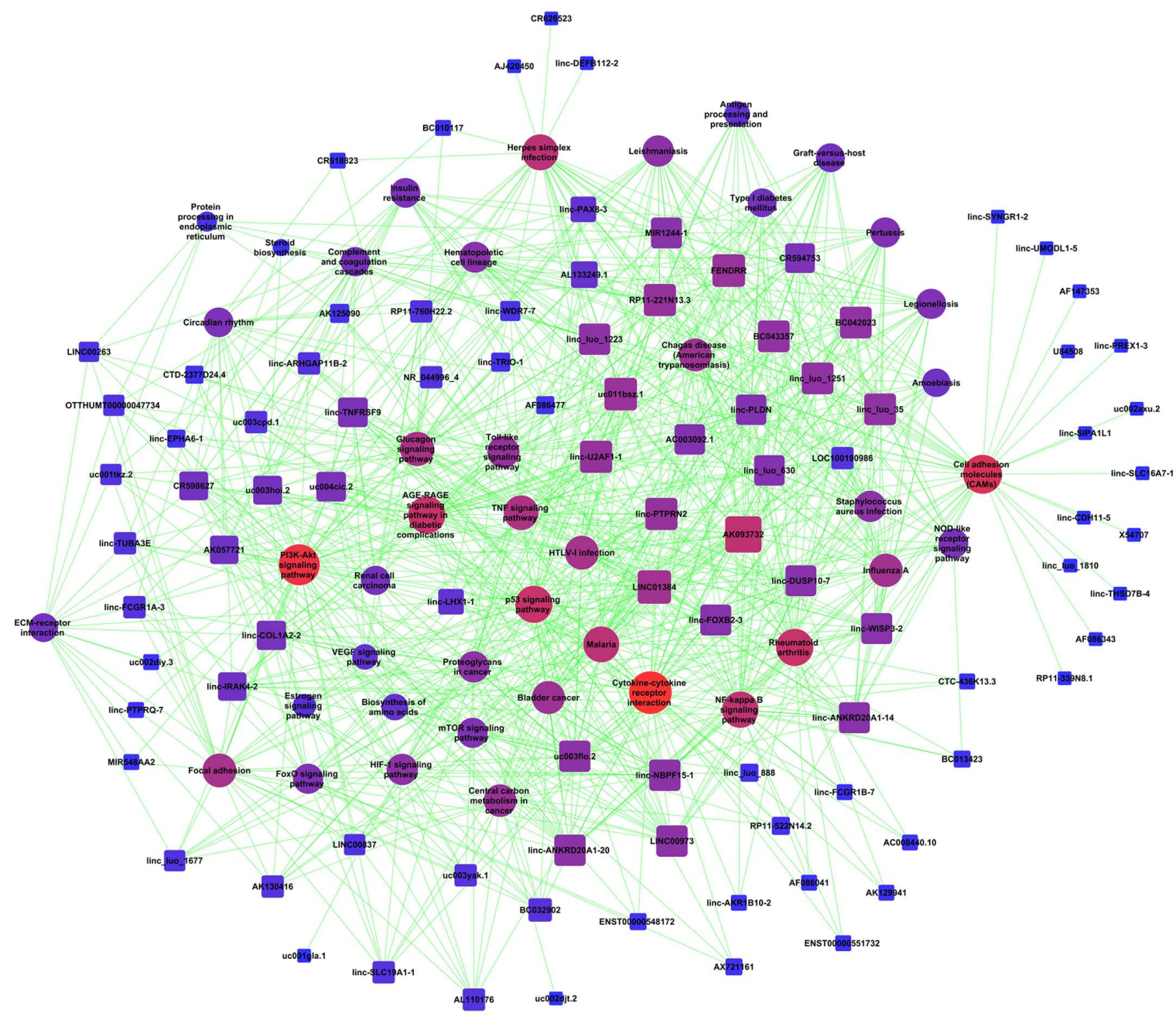

Figure 7. IncRNA target pathway network in AGPS-silenced U251 cells. The regulatory core pathways of differential lncRNAs were identified using a lncRNA-gene-pathway network, indicating the association between lncRNAs and pathways in AGPS-silenced glioma U251 cells. Red represents the higher Degree values, blue represents the lower Degree values and purple represents the middle Degree values. AGPS, alkylglycerone phosphate synthase; IncRNA, long non-coding RNA.

promoted the apoptosis of human brain microvascular endothelial cells (27). Other previous studies have also reported that FENDRR served an important regulatory role in numerous types of malignant tumor, such as human lung, colon, breast and liver cancer (28-30). For example, FENDRR reduced the stem cell characteristics of non-small cell lung cancer by inhibiting the ELAV-like protein 1/P-glycoprotein axis; and FENDRR was also discovered to inhibit Sox4 protein expression, which slowed the progression of colon cancer (31). However, to the best of our knowledge, there has been no previous research conducted on the role of FENDRR in glioma. In the present study, the expression levels of FENDRR in the AGPS-silenced groups were significantly upregulated compared with the control group, indicating that the expression levels of FENDRR may be negatively associated with the degree of glioma malignancy. However, the role of FENDRR in glioma cells and its related mechanisms requires further investigation.

It has been revealed that the expression levels of the lncRNA-PTPRN2 in the brains of mice treated with a neuroprotective preparation of trans-resveratrol were significantly downregulated compared with mice fed the standard diet (32).
Notably, a previous study suggested that the lncRNA-PTPRN2, which had a pivotal position in the pathway regulatory network, may be involved in the regulation of cell adhesion molecules by regulating the expression levels of intercellular adhesion molecule- 1 and signal transduction pathways, such as advanced glycation end products/receptor for advanced glycosylation end products, NF- $\mathrm{\kappa B}$ and tumor necrosis factor (33). It was reported that also found that lncRNA AK093732 was an important site and highest Degree. In a previous study, lncRNA AK093732 was increased in laryngeal squamous cell carcinoma and considered a potential biomarker (34). However, further research is required to identify the regulatory mechanisms of PTPRN2 and AK093732 in these signaling pathways.

In conclusion, the present study analyzed the association and role of AGPS with the expression levels of lncRNAs and mRNAs in glioma. It was discovered that the identified differentially expressed lncRNAs and co-expressed mRNAs may serve important biological roles in the development, progression and metastasis of gliomas. Furthermore, the pathway networks identified also provide a reference and direction for further studies into the regulatory mechanisms of important lncRNAs in glioma and their associated signaling pathways. 


\section{Acknowledgements}

Not applicable.

\section{Funding}

The present study was supported by the Special Program of Talent Development for Excellent Youth Scholars in Tianjin, China (grant no. TJTZJH-QNBJRC-2-9), the Natural Science Foundation of Tianjin (grant no. 16JCQNJC11500) and the Science and Technology Development Fund of Bengbu Medical College (grant no. BYKF1783).

\section{Availability of data and materials}

All data generated or analyzed during this study are included in this published article.

\section{Authors' contributions}

YZ was responsible for the conception and design of the study. LC and WZ were responsible for acquisition of data. $\mathrm{LH}$ and LJ were responsible for data interpretation. LQ was responsible for cellular and molecular experiments. All authors read and approved the final manuscript.

\section{Ethics approval and consent to participate}

Not applicable.

\section{Patient consent for publication}

Not applicable.

\section{Competing interests}

The authors declare that they have no competing interests.

\section{References}

1. Ferris SP, Hofmann JW, Solomon DA and Perry A Characterization of gliomas: From morphology to molecules. Virchows Arch 2: 257-269, 2017.

2. Benjamin Daniel I, Cravatt Benjamin F and Nomura Daniel K: Global profiling strategies for mapping dysregulated metabolic pathways in cancer. Cell Metab 5: 565-577, 2012.

3. Piano V, Benjamin DI, Valente S, Nenci S, Marrocco B, Mai A, Aliverti A, Nomura DK and Mattevi A: Discovery of inhibitors for the ether lipid-generating enzyme AGPS as anti-cancer agents. ACS Chem Biol 11: 2589-2597, 2015.

4. Benjamin DI, Cozzo A, Ji X, Roberts LS, Louie SM, Mulvihill MM, Luo K and Nomura DK: Ether lipid generating enzyme AGPS alters the balance of structural and signaling lipids to fuel cancer pathogenicity. Proc Natl Acad Sci USA 110: 14912-14917, 2013.

5. Hou S, Tan J, Yang B, He L and Zhu Y: Effect of alkylglycerone phosphate synthase on the expression profile of circRNAs in the human thyroid cancer cell line FRO. Oncol Lett 15: 7889-7899, 2018

6. Stazi G, Battistelli C, Piano V, Mazzone R, Marrocco B, Marchese S, Louie SM, Zwergel C, Antonini L, Patsilinakos A, et al: Development of alkyl glycerone phosphate synthase inhibitors: Structure-activity relationship and effects on ether lipids and epithelial-mesenchymal transition in cancer cells. Eur J Med Chem 163: 722-735, 2019.

7. Zhu Y, Zhu L, Lu L, Zhang L, Zhang G, Wang Q and Yang P. Role and mechanism of the alkylglycerone phosphate synthase in suppressing the invasion potential of human glioma and hepatic carcinoma cells in vitro. Oncol Rep 32: 431-436, 2014.
8. Jarroux J, Morillon A and Pinskaya M: History, discovery, and classification of lncRNAs. Adv Exp Med Biol 1008: 1-46, 2017.

9. Jiang X, Yan Y, Hu M, Chen X, Wang Y, Dai Y, Wu D, Wang Y, Zhuang $\mathrm{Z}$ and $\mathrm{Xia} \mathrm{H}$ : Increased level of H19 long noncoding RNA promotes invasion, angiogenesis, and stemness of glioblastoma cells. J Neurosurg 2016: 129-136, 2016.

10. Lim W and Kim HS: Exosomes as therapeutic vehicles for cancer. Tissue Eng Regen Med 16: 213-223, 2019.

11. Livak KJ and Schmittgen TD: Analysis of relative gene expression data using real-time quantitative PCR and the 2(-Delta Delta C(T)) method. Methods 25: 402-408, 2001.

12. Noman MZ, Hasmim M, Messai Y, Terry S, Kieda C, Janji B and Chouaib S: Hypoxia: A key player in antitumor immune response. A review in the theme: Cellular responses to hypoxia. Am J Physiol Cell Physiol 309: C569-C579, 2015.

13. Pickup MW, Mouw JK and Weaver VM: The extracellular matrix modulates the hallmarks of cancer. EMBO Rep 15: 1243-1253, 2014.

14. Soni H, Bode J, Nguyen CDL, Puccio L, Neßling M, Piro RM, Bub J, Phillips E, Ahrends R, Eipper BA, et al: PERK-mediated expression of peptidylglycine $\alpha$-amidating monooxygenase supports angiogenesis in glioblastoma. Oncogenesis 9: 18, 2020.

15. Wood R and Snyder F: Characterization and identification of glyceryl ether diesters present in tumor cells. J Lipid Res 8: 494-500, 1967.

16. Jaffrès PA, Gajate C, Bouchet AM, Couthon-Gourvès H, Chantôme A, Potier-Cartereau M, Besson P, Bougnoux P, Mollinedo F and Vandier C: Alkyl ether lipids, ion channels and lipid raft reorganization in cancer therapy. Pharmacol Ther 165: 114-131, 2016.

17. Li Y,Han X, Feng $H$ and Han J: Long noncoding RNA OIP5-AS1 in cancer. Clin Chim Acta 499: 75-80, 2019.

18. Shen J, Cao B, Wang Y, Ma C, Zeng Z, Liu L, Li X, Tao D, Gong $\mathrm{J}$ and Xie D: Hippo component YAP promotes focal adhesion and tumour aggressiveness via transcriptionally activating THBS1/FAK signalling in breast cancer. J Exp Clin Cancer Res 37: 175, 2018.

19. Wu P, Wang Y, Wu Y, Jia Z, Song Y and Liang N: Expression and prognostic analyses of ITGA11, ITGB4 and ITGB8 in human non-small cell lung cancer. PeerJ 7: e8299, 2019.

20. Jeong BY, Cho KH, Jeong KJ, Park YY, Kim JM, Rha SY, Park CG, Mills GB, Cheong JH and Lee HY: Rab25 augments cancer cell invasiveness through a $\beta 1$ integrin/EGFR/VEGF-A/Snail signaling axis and expression of fascin. Exp Mol Med 50: e435, 2018.

21. Zeng B, Zhou M, Wu H and Xiong Z: SPP1 promotes ovarian cancer progression via Integrin $\beta 1 / \mathrm{FAK} / \mathrm{AKT}$ signaling pathway. Onco Targets Ther 11: 1333-1343, 2018.

22. Rhead B, Shao X, Quach H, Ghai P, Barcellos LF and Bowcock AM: Global expression and $\mathrm{CpG}$ methylation analysis of primary endothelial cells before and after TNFa stimulation reveals gene modules enriched in inflammatory and infectious diseases and associated DMRs. PLoS One 15: e0230884, 2020

23. Ni M, Liu X, Meng Z, Liu S, Jia S, Liu Y, Zhou W, Wu J, Zhang J, Guo S, et al: A bioinformatics investigation into the pharmacological mechanisms of javanica oil emulsion injection in non-small cell lung cancer based on network pharmacology methodologies. BMC Complement Med Ther 20: 174, 2020.

24. Long Y, Wang X, Youmans DT and Cech TR: How do lncRNAs regulate transcription? Sci Adv 3: eaao2110, 2017.

25. Abedini P, Fattahi A, Agah S, Talebi A, Beygi AH, Amini SM, Mirzaei A and Akbari A: Expression analysis of circulating plasma long noncoding RNAs in colorectal cancer: The relevance of lncRNAs ATB and CCAT1 as potential clinical hallmarks. J Cell Physiol 234: 22028-22033, 2019.

26. Liang WC, Ren JL, Wong CW, Chan SO, Waye MM, Fu WM and Zhang JF: LncRNA-NEF antagonized epithelial to mesenchymal transition and cancer metastasis via cis-regulating FOXA2 and inactivating Wnt/ $\beta$-catenin signaling. Oncogene 37: 1445-1456, 2018.

27. Wang B, Xian J, Zang J, Xiao L, Li Y, Sha M and Shen M: Long non-coding RNA FENDRR inhibits proliferation and invasion of hepatocellular carcinoma by down-regulating glypican-3 expression. Biochem Biophys Res Commun 509: 143-147, 2019.

28. Zhang G, Han G, Zhang X, Yu Q, Li Z, Li Z and Li J: Long non-coding RNA FENDRR reduces prostate cancer malignancy by competitively binding miR-18a-5p with RUNX1. Biomarkers 23: 435-445, 2018. 
29. Liu J and Du W: LncRNA FENDRR attenuates colon cancer progression by repression of SOX4 protein. Onco Targets Ther 12: 4287-4295, 2019; Li Y,Zhang W, Liu P, Xu Y, Tang L, Chen W and Guan X: Long non-coding RNA FENDRR inhibits cell proliferation and is associated with good prognosis in breast cancer. Onco Targets Ther 11: 1403-1412, 2018.

30. Xu R and Han Y: Long non-coding RNA FOXF1 adjacent non-coding developmental regulatory RNA inhibits growth and chemotherapy resistance in non-small cell lung cancer. Arch Med Sci 15: 1539-1546, 2019.

31. Gong $\mathrm{F}$, Dong $\mathrm{D}$, Zhang $\mathrm{T}$ and $\mathrm{Xu} \mathrm{W}$ : Long non-coding RNA FENDRR attenuates the stemness of non-small cell lung cancer cells via decreasing multidrug resistance gene 1 (MDR1) expression through competitively binding with RNA binding protein HuR. Eur J Pharmacol 853: 345-352, 2019.
32. Navarro G, Martínez-Pinilla E, Sánchez-Melgar A, Ortiz R, Noé V, Martín M, Ciudad C and Franco R: A genomics approach identifies selective effects of trans-resveratrol in cerebral cortex neuron and glia gene expression. PLoS One 12: e0176067, 2017.

33. Lee EJ, Rath P, Liu J, Ryu D, Pei L, Noonepalle SK, Shull AY, Feng Q, Litofsky NS, Miller DC, et al: Identification of global DNA methylation signatures in glioblastoma-derived cancer stem cells. J Genet Genomics 42: 355-371, 2015.

34. Shen Z, Li Q, Deng H, Lu D, Song H and Guo J: Long non-coding RNA profiling in laryngeal squamous cell carcinoma and its clinical significance: Potential biomarkers for LSCC. PLoS One 9: e108237, 2014.

This work is licensed under a Creative Commons Attribution-NonCommercial-NoDerivatives 4.0 International (CC BY-NC-ND 4.0) License. 